\title{
AVALIAÇÃO DOS ÍNDICES DE CONFORTO TÉRMICO, PARÂMETROS FISIOLÓGICOS E GRADIENTE TÉRMICO DE OVINOS NATIVOS
}

\author{
NEYLA L. RIBEIRO ${ }^{1}$, DERMEVAL A. FURTADO ${ }^{2}$, ARIOSVALDO N. MEDEIROS ${ }^{3}$, \\ MARIA N. RIBEIRO ${ }^{4}$, REGINA C.B. SILVA ${ }^{4}$, CICILIA M. S. SOUZA ${ }^{5}$
}

\begin{abstract}
RESUMO: Esta pesquisa teve o objetivo de determinar os índices de conforto térmico em instalações para ovinos, analisar os parâmetros fisiológicos e o gradiente térmico de quatro grupos genéticos, na região semiárida paraibana. Foram utilizados 40 animais, dez por grupo genético, que foram: Cariri, Morada Nova, Barriga Negra e Cara Curta, todas fêmeas, alojadas aleatoriamente em quatro apriscos. Os índices ambientais dentro das instalações, principalmente das 11 às $15 \mathrm{~h}$, ficaram acima da zona de conforto térmico para ovinos adultos, com exceção da umidade relativa do ar, que ficou com média diária de $67,5 \%$. A temperatura retal dos animais esteve dentro da faixa normal, sendo o grupo genético Morada Nova o que apresentou menor valor $\left(38,6^{\circ} \mathrm{C}\right)$. A frequência respiratória dos animais em cada grupo genético ficou acima do valor recomendado, porém o Morada Nova apresentou o menor valor $\left(43,5 \mathrm{mov} \mathrm{min}^{-1}\right)$; em contrapartida, o Cariri e o Barriga Negra apresentaram os maiores valores $\left(48,0\right.$ e 47,3 $\mathrm{mov} \mathrm{min}^{-1}$, respectivamente). Os animais com pelagem mais escura e de maior porte, como os da raça Cariri, apresentaram maior gradiente térmico entre a temperatura do ar e a temperatura superficial. Os animais apresentaram alto índice de tolerância ao calor, ou seja, bem adaptados ao ambiente tropical, podendo o grupo genético Cariri ser caracterizado como o menos adaptado e o Morada Nova considerado o mais bem adaptado às condições experimentais.
\end{abstract}

PALAVRAS-CHAVE: ambiência, conforto térmico, animais nativos.

\section{ASSESSMENT OF THERMAL COMFORT INDEXES, PHYSIOLOGICAL PARAMETERS AND THERMAL GRADIENT OF NATIVE SHEEP}

ABSTRACT: The objective of this research was to determine the thermal comfort indexes for sheep raising installations, analyze the physiological parameters and the thermal gradient of four sheep genetic groups in the semi-arid region of the state of Paraiba, Brazil. Forty animals were used, being ten individuals per genetic group; the groups were: 'Cariri', 'Morada Nova', 'Barriga Negra' and 'Cara Curta'. All of them females and randomly housed in four adequate installations. The environmental indexes inside the lodging, mainly from 11 a.m. to 13 p.m., remained above the thermal comfort zone for adult sheep, except for the relative humidity, that had a daily average of $67.5 \%$. The rectal temperature of the animals was kept within the normal range, except for the genetic group 'Morada Nova' that presented the lowest values $\left(38.6^{\circ} \mathrm{C}\right)$. The respiration frequency for each of the genetic group was above the normal value; with the 'Morada Nova' once again

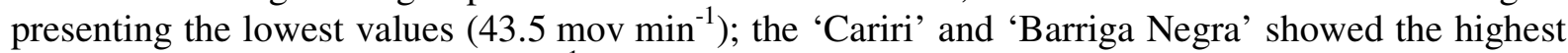
frequency (48.0 and $47.3 \mathrm{mov} \mathrm{min}^{-1}$, respectively). Animals with darker hair color and larger size, such as those belonging to the genetic group 'Cariri', presented a higher thermal gradient between air and body surface temperatures. Animals showed high tolerance to heat, in other words, they are well adapted to the tropical environment, except for the genetic group 'Cariri' that was the least adapted. The genetic group 'Morada Nova' was considered the best adapted group for the experimental conditions.

KEYWORDS: environment, thermal comfort, native animals.

\footnotetext{
1 Zootecnista, M.Sc. em Engenharia Agrícola, Fone: (0XX83) 9982.2736, neilalr@yahoo.com.br

2 Prof. Adjunto, Departamento de Engenharia Agrícola, UFCG, Fone (0XX83) 3310.1486, dermeval@ deag.ufcg.edu.br

${ }^{3}$ Prof. Adjunto, Departamento de Zootecnia, UFPB, Areia - PB.

5 Zootecnista, aluna do curso de Doutorado em Zootecnia, UFPB, Areia - PB.

${ }^{6}$ Zootecnista, aluna do curso de Doutorado em Zootecnia, UFPB, Areia - PB.

Recebido pelo Conselho Editorial em: 12-9-2006

Aprovado pelo Conselho Editorial em: 6-10-2008
} 


\section{INTRODUÇÃO}

Ao analisar a adaptabilidade dos animais à região semiárida nordestina, principalmente quando se trabalha com raças nativas, é fundamental que o fator climático seja levado em consideração, pela sua vulnerabilidade às alterações do clima, com períodos irregulares de chuva e secas prolongadas, que são intensificados pelas elevadas temperaturas do ar, altos níveis de insolação e evaporação e, durante o período seco, pela baixa umidade relativa do ar (SANTOS et al., 2005). Os ovinos nativos são descendentes de animais trazidos pelos colonizadores portugueses e, mais tarde, pelos espanhóis e franceses, e representam grande parte do rebanho brasileiro.

De acordo com MONTY JÚNIOR et al. (1991), para obter sucesso em uma criação, devem-se escolher raças ou linhagens que sejam adaptadas às condições da região. $\mathrm{O}$ aumento da temperatura ambiente acima daquela considerada crítica máxima para o animal pode desencadear reações ou respostas fisiológicas, tais como: aumento da temperatura retal, temperatura da superfície da pele e frequência respiratória, ocorrendo diminuição do nível de produção e ingestão de alimentos (LU, 1989).

Segundo BACARI JÚNIOR (1990), as avaliações de adaptabilidade dos animais aos ambientes quentes podem ser realizadas por meio de testes de adaptabilidade fisiológica ou de tolerância ao calor. Para BIANCA \& KUNZ (1978), a temperatura retal e a frequência respiratória são consideradas as melhores variáveis fisiológicas para estimar a tolerância dos animais ao clima quente.

De acordo com CUNNINGHAN (2004), a temperatura retal normal em ovinos varia de 38,5 a $39,9{ }^{\circ} \mathrm{C}$, e vários fatores são capazes de causar variações na temperatura corporal, entre os quais: idade, sexo, estação do ano, período do dia, exercício, ingestão e digestão de alimentos. Segundo CESAR et al. (2004) e OLIVEIRA et al. (2005), a temperatura retal dos ovinos é afetada durante o dia, e os animais mostram temperatura retal menor no período da manhã, comparados com o período da tarde. Esses dados têm implicações práticas relevantes, pois indicam que, no final da tarde e à noite, as condições de manutenção da homeotermia são mais favoráveis para os ovinos. No decorrer do dia, com o aumento da temperatura ambiente, os animais entram em processo de hipertermia, com redução do apetite e, consequentemente, redução na ingestão de alimentos.

Segundo REECE (1996), a frequência respiratória em ovinos varia entre 20 a 34 mov min ${ }^{-1}$, sendo excelente indicador do estado de saúde ou de conforto térmico dos animais, mas deve ser adequadamente interpretada, uma vez que pode ser influenciada pela espécie, idade, exercícios, excitação e fatores ambientais. Assim, se ocorrer frequência respiratória alta e o animal for eficiente em eliminar o calor, poderá não ocorrer o estresse calórico (BERBIGIER, 1989). Segundo McDOWELL (1989), a frequência respiratória alta pode ser eficiente maneira de perder calor por curtos períodos, mas, caso mantido por várias horas, poderá resultar em sérios problemas para os animais.

A pele protege o organismo do calor e do frio, e sua temperatura depende, principalmente, das condições ambientais, como temperatura, umidade e vento, e das condições fisiológicas, como a vascularização e a evaporação do suor. Em temperaturas mais amenas, os animais dissipam calor sensível para o ambiente através da pele, por radiação, por condução e por convecção. Quando os animais estão sob estresse pelo calor, as perdas sensíveis são diminuídas, e o principal processo de perda de calor é o latente, ou seja, pela evaporação e respiração (SILVA, 2000).

Os animais também utilizam outros processos para manter a homeotermia, como a vasodilatação periférica, que aumenta o fluxo sanguíneo para a superfície corporal, aumentando a temperatura da superfície animal (CHIMINEAU, 1993). Segundo HABEEB et al. (1992) essa vasodilatação facilita a troca de calor do animal com o meio ambiente por processos não-evaporativos (condução, convecção e radiação), mas que esse processo, para se tornar eficaz, depende do gradiente térmico entre o corpo do animal e a temperatura ambiente. 
O objetivo do trabalho foi analisar os índices de conforto térmico, os parâmetros fisiológicos e o índice de tolerância ao calor (adaptabilidade) de quatro grupos genéticos de ovinos, quais sejam: Cariri, Cara Curta, Morada Nova e Barriga Negra, na região semiárida paraibana.

\section{MATERIAL E MÉTODOS}

O experimento foi realizado na Estação Experimental de São João do Cariri, pertencente à Universidade Federal da Paraíba, no município de São João do Cariri - PB, no período de março a maio de 2005, no total de 74 dias, sendo 14 de adaptação dos animais. Segundo a classificação climática de Thornthwaite, o clima da região é semiárido $(-60<\operatorname{Im}<-40)$, com precipitação média anual de $450 \mathrm{~mm}$, latitude de $7^{\circ} 25^{\prime} \mathrm{S}$ e longitude de $36^{\circ} 30^{\prime} \mathrm{W}$, altitude média de 450 a $500 \mathrm{~m}$, temperatura média anual de $26{ }^{\circ} \mathrm{C}$ e umidade relativa média do ar de $63 \%$.

Foram utilizadas 40 fêmeas, dez por grupo genético: Cariri, Barriga Preta, Cara Curta e Morada Nova (Figuras 1; 2; 3 e 4), com massa média inicial de 37,23; 40,57; 35,88 e 33,29 kg, respectivamente, distribuídas aleatoriamente em quatro apriscos, medindo $52,5 \mathrm{~m}^{2}$. Cada aprisco era dividido em dez baias de $3,75 \mathrm{~m}^{2}$ cada e possuía corredor central de 2,0 m de largura, pé-direito de $2,8 \mathrm{~m}$, com pilares de madeira e orientação do eixo principal no sentido leste-oeste e divisória lateral com tela de arame galvanizado. As baias tinham o piso de terra batida e cobertura de telhas cerâmicas.

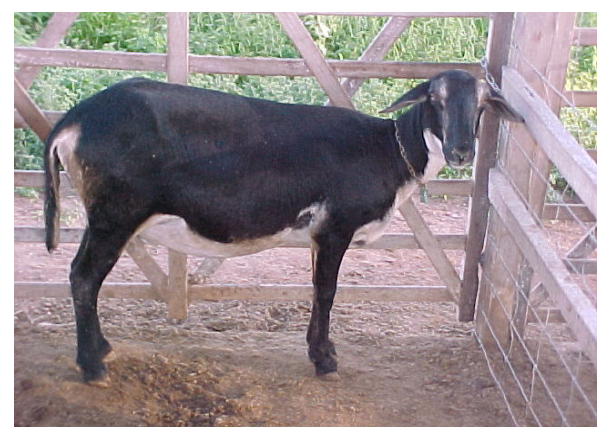

FIGURA 1. Grupo genético Cariri. Genetic group Cariri.

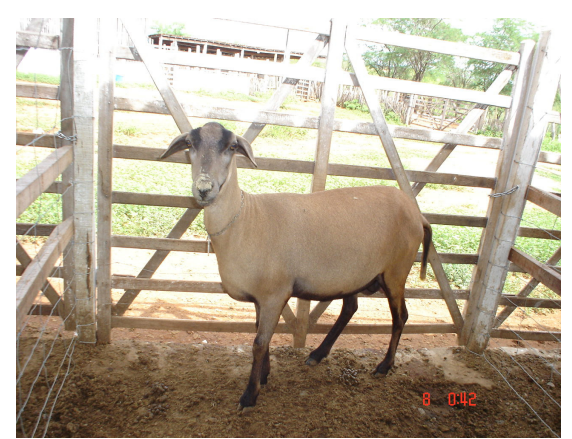

FIGURA 3. Grupo genético Barriga Negra. Genetic group Barriga Negra.

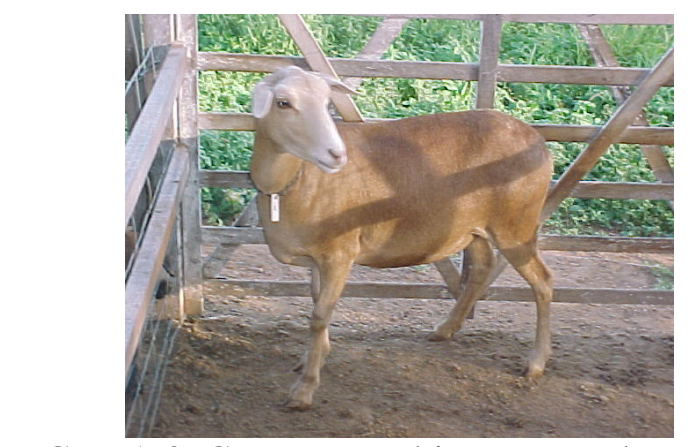

FIGURA 2. Grupo genético Morada Nova. Genetic group Morada Nova.

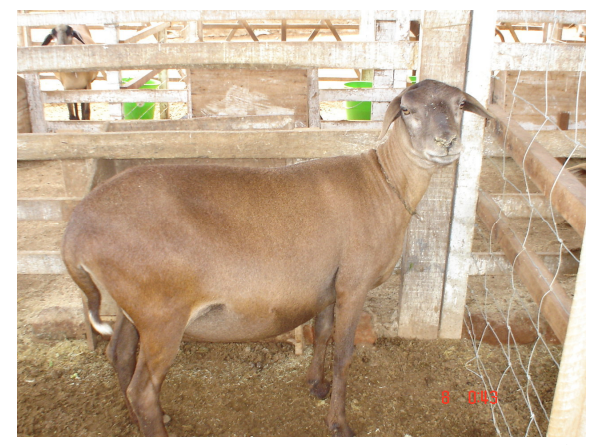

FIGURA 4. Grupo genético Cara Curta. Genetic group Cara Curta.

Durante o período experimental, foram coletadas, a cada duas horas, no intervalo das 7 às $17 \mathrm{~h}$, a temperatura do bulbo seco (TA), temperatura de bulbo úmido (Tbu), temperatura do globo negro (Tgn) e velocidade do vento. Foram coletadas, também, às 16 h, a temperatura máxima (Tmáx) e a temperatura mínima (Tmín). Para a coleta dos dados, utilizaram-se termômetros de bulbo seco, termômetros de bulbo úmido e termômetro de máxima e mínima. Todos os termômetros utilizados eram de mercúrio, em vidro, com resolução de $1,0{ }^{\circ} \mathrm{C}$. As temperaturas de globo negro foram determinadas por meio de termômetro de globo negro, com diâmetro de $0,15 \mathrm{~m}$, sendo no seu interior instalado, como sensor, um termômetro de mercúrio, em vidro, com resolução de $1,0{ }^{\circ} \mathrm{C}$. A 
velocidade do vento foi determinada por meio de anemômetro digital de hélice, com resolução de $0,01 \mathrm{~m} \mathrm{~s}^{-1}$. Os equipamentos foram localizados no centro de massa dos animais, ou seja, a $0,70 \mathrm{~m} \mathrm{de}$ altura.

A partir dos valores obtidos para as variáveis climatológicas, determinaram-se a umidade relativa do ar (UR) e o índice de temperatura de globo negro e umidade (ITGU) e a carga térmica de radiação (CTR). O ITGU foi obtido pela expressão citada por BUFFINGTON et al. (1977), e a CTR foi calculada pela expressão citada por ESMAY, 1969 (eq.(1):

$$
\text { ITGU }=\mathrm{T}_{\mathrm{gn}}+0,36 \mathrm{~T}_{\mathrm{d}}-330,08
$$

em que,

Tgn - temperatura do globo negro, e

$\mathrm{Td}$ - temperatura do ponto de orvalho, ambas expressas em $\mathrm{K}$.

em que,

$$
\mathrm{CTR}=\sigma(\mathrm{TRM})^{4}
$$

CTR - W m ${ }^{-2}$

$\sigma$ - constante de Stefan-Boltzman $\left(5,6710^{-8} \mathrm{~W} \mathrm{~m}^{-2} \mathrm{~K}^{-4}\right)$, e

TRM - temperatura radiante média, expressa em $\mathrm{K}$.

A TRM é a temperatura de uma circunvizinhança, considerada uniformemente negra, para eliminar o efeito da reflexão, com a qual o corpo (globo negro) troca tanta quantidade de energia quanto a do ambiente considerado (BOND et al., 1954). A TRM foi obtida pela eq.(3):

em que,

$$
\mathrm{TRM}=100\left[2,51 \mathrm{v}^{1 / 2}\left(\mathrm{~T}_{\mathrm{gn}}-\mathrm{T}_{\mathrm{a}}\right)+\left(\mathrm{T}_{\mathrm{gn}} / 100\right)^{4}\right]^{1 / 4}
$$

TRM - K;

$\mathrm{V}$ - velocidade do vento, $\mathrm{m} \mathrm{s}^{-1}$, e

$\mathrm{Ta}$ - temperatura ambiente, $\mathrm{K}$.

As variáveis fisiológicas analisadas foram a temperatura retal (TR), a frequência respiratória (FC) e a temperatura superficial do pelame (TS). As variáveis foram coletadas durante quatro dias por semana, às 9 e às $15 \mathrm{~h}$. Para a obtenção da TR, foi introduzido, diretamente no reto do animal, um termômetro clínico veterinário, com profundidade de $5 \mathrm{~cm}$, permanecendo durante 2 minutos, sendo o resultado da leitura expresso em graus Celsius. Para a obtenção da FR, empregou-se um estetoscópio flexível, na região torácica direita, perto das primeiras costelas, contando-se o número de movimentos durante 20 segundos, sendo o valor obtido multiplicado por três, determinando os movimentos por minuto ( $\mathrm{mov} \mathrm{min}^{-1}$ ).

A TS foi coletada por meio de termômetro infravermelho, com mira laser, na cabeça, no costado e nas pernas dos animais, e depois se utilizou da média dessas três temperaturas para o cálculo da TS. Com a média da TS, foram calculados os gradientes térmicos entre a TR e a TS, e entre a TS e a TA (utilizando-se da média das 9 e das 15 h, para a TR e TA, respectivamente).

Para a realização do índice de tolerância ao calor (ITC), adaptou-se a metodologia proposta por BACARI JÚNIOR et al. (1986), em que os animais foram mantidos durante duas horas na sombra (das 12 às $14 \mathrm{~h}$ ); das 14 às $15 \mathrm{~h}$, foram expostos à radiação solar direta, sendo, após esse tempo, coletada a primeira temperatura retal $\left(\mathrm{TR}_{1}\right)$; em seguida, os animais foram conduzidos à sombra, permanecendo em repouso por uma hora para, em seguida, ser tomada a segunda temperatura retal $\left(\mathrm{TR}_{2}\right)$. $\mathrm{O}$ teste foi repetido por seis dias durante o período experimental.

$$
\mathrm{ITC}=10-\left(\mathrm{TR}_{2}-\mathrm{TR}_{1}\right)
$$

Utilizou-se de delineamento inteiramente casualizado, em fatorial 4 x 2, sendo quatro grupos genéticos (Cariri, Barriga Negra, Morada Nova e Cara Curta) e dois turnos (manhã e tarde). 
Os dados TR, FR, TS, ITGU, CTR, UR, Tgn e TA foram submetidos à análise de variância pelo procedimento GLM do SAS (1999), utilizando-se do modelo matemático descrito na eq.(5), sendo as médias comparadas pelo teste de Tukey, a 5\% de probabilidade.

Yijkl $=\mu+R i+T j+R T k+€ i j k l$

em que,

Yijkl - variável estudada;

$\mu$ - média comum a todas as observações;

$\mathrm{Ri}$ - efeito de raça;

$\mathrm{Tj}$ - efeito de turno;

RT - efeito da interação raça $x$ turno, e

$€ \mathrm{ijkl}$ - erro comum a todas as observações $\left(0, \sigma^{2}\right)$.

\section{RESULTADOS E DISCUSSÃO}

O resumo da análise de variância das variáveis ambientais temperatura do ar (TA), umidade relativa do ar (UR), temperatura de globo negro (Tgn), índice de temperatura de globo negro e umidade (ITGU) e carga térmica radiante (CTR) está apresentado na Tabela 1, na qual se observa que todas as variáveis tiveram efeito significativo para os horários $(\mathrm{p}<0,01)$.

TABELA 1. Resumo da análise de variância das variáveis ambientais: temperatura do ar (TA), umidade relativa (UR), temperatura de globo negro (Tgn), índice de temperatura de globo negro e umidade (ITGU), carga térmica radiante (CTR). Summary of the analysis of variance for the air temperature (AT), relative humidity (RH), black globe temperature $\left(T_{b g}\right)$, index of the black globe temperature and humidity (IBGH), radiant heat load (RHL).

\begin{tabular}{ccccccc}
\hline Fonte de & \multirow{2}{*}{ GL } & \multicolumn{5}{c}{ Quadrados Médios } \\
\cline { 3 - 6 } Variação & & TA & UR & Tgn & ITGU & CTR \\
\hline Hora & 5 & $619,946^{* *}$ & $11.271,21^{* *}$ & $706,394^{* *}$ & $685,246^{* *}$ & $52.257,312^{* *}$ \\
Erro & 550 & 4,488 & 123,4097 & 6,9172 & 6,6409 & 890,9089 \\
$\mathrm{R}^{2}$ & & 0,56 & 0,45 & 0,48 & 0,48 & 0,35 \\
C.V. $(\%)$ & & 7,56 & 16,26 & 8,70 & 3,26 & 5,84 \\
\hline
\end{tabular}

** P<0,01; C.V.(\%) - coeficiente de variação

As médias das variáveis ambientais Tmáx, Tmín, TA, UR, Tgn, ITGU e CTR estão apresentadas na Tabela 2. Observa-se que a Tmáx esteve fora da zona de conforto térmico (ZCT) citada por BAÊTA \& SOUZA (1997), para ovinos adultos, que é de $30{ }^{\circ} \mathrm{C}$, e a Tmín permaneceu dentro da ZCT. OLIVEIRA et al. (2005), em trabalhos na região semiárida, com ovinos Santa Inês, citam temperatura máxima de $31,3{ }^{\circ} \mathrm{C}$, semelhante à encontrada neste trabalho, sendo a Tmín inferior a $20,3{ }^{\circ} \mathrm{C}$.

Para a TA, os valores diferiram estatisticamente $(\mathrm{P}>0,05)$, com exceção dos valores das 13 e das $15 \mathrm{~h}$ e dos valores da TA no horário das 7; 9; 11 e 17 h, que estiveram dentro da ZCT. A UR nos diferentes horários, com exceção das $7 \mathrm{~h}$, esteve dentro da faixa de conforto térmico para os animais, que, segundo BAETA \& SOUZA (1997), deve estar entre 50 e $80 \%$. Às 13 e às 15 h, os valores da UR não diferiram entre si $(\mathrm{P}>0,05)$. Os valores de Tgn diferiram estatisticamente $(\mathrm{P}<0,05)$ entre si, com exceção dos valores das 13 e das $15 \mathrm{~h}$, sendo o valor das $15 \mathrm{~h}\left(33,4{ }^{\circ} \mathrm{C}\right)$ o mais elevado e ocorreu crescimento ao longo do dia, até as $15 \mathrm{~h}$, quando começou a decrescer. 
TABELA 2. Médias das variáveis ambientais temperatura máxima (Tmáx), temperatura mínima (Tmín), temperatura do ar (TA), umidade relativa (UR), temperatura de globo negro (Tgn), índice de temperatura de globo negro e umidade (ITGU) e carga térmica radiante (CTR). Average values of environmental variables: maximum temperature (Tmax), minimum temperature (Tmin), air temperature (AT), relative humidity $(\mathrm{RH})$, black globe temperature $\left(\mathrm{T}_{\mathrm{bg}}\right)$, index of the black globe temperature and humidity (IBGH) and radiant heat load (RHL).

\begin{tabular}{|c|c|c|c|c|c|c|c|}
\hline \multirow{2}{*}{ Horários } & \multicolumn{6}{|c|}{ Variáveis Ambientais } & \multirow[b]{2}{*}{$\operatorname{CTR}\left(\mathrm{W} \mathrm{m}^{-2}\right)$} \\
\hline & Tmáx $\left({ }^{\circ} \mathrm{C}\right)$ & Tmín $\left({ }^{\circ} \mathrm{C}\right)$ & $\mathrm{TA}\left({ }^{\circ} \mathrm{C}\right)$ & UR $(\%)$ & $\operatorname{Tgn}\left({ }^{\circ} \mathrm{C}\right)$ & ITGU & \\
\hline 7 & & & $23,7 \mathrm{a}$ & $85,9 \mathrm{a}$ & $26,3 \mathrm{a}$ & $75,1 \mathrm{a}$ & $483,8 \mathrm{a}$ \\
\hline 9 & & & $26,7 \mathrm{~b}$ & $70,6 \mathrm{~b}$ & $29,2 \mathrm{~b}$ & $77,9 \mathrm{~b}$ & $505,2 \mathrm{~b}$ \\
\hline 11 & & & $28,7 \mathrm{c}$ & $64,2 \mathrm{c}$ & $31,3 \mathrm{c}$ & $80,1 \mathrm{c}$ & $519,8 \mathrm{c}$ \\
\hline 13 & & & $30,2 \mathrm{~d}$ & $57,4 \mathrm{~d}$ & $32,7 \mathrm{~d}$ & $81,3 \mathrm{~d}$ & $529,9 \mathrm{~d}$ \\
\hline 15 & & & $30,1 \mathrm{~d}$ & $59,1 \mathrm{~d}$ & $33,4 \mathrm{~d}$ & $82,1 \mathrm{~d}$ & $543,6 \mathrm{e}$ \\
\hline 17 & 31,3 & 22,9 & $27,5 \mathrm{e}$ & $67,8 \mathrm{e}$ & $29,2 \mathrm{~b}$ & $77,9 \mathrm{~b}$ & 491,5 af \\
\hline Média & & & 27,8 & 67,5 & 30,4 & 79,06 & 512,3 \\
\hline
\end{tabular}

*Médias nas colunas, seguidas de letras diferentes, diferem entre si, pelo teste de Tukey, a 5\% de probabilidade.

Quanto ao ITGU, observa-se que houve diferença significativa entre os horários analisados, sendo semelhantes nos horários das 9 e $17 \mathrm{~h}$ e das 13 e $15 \mathrm{~h}$. O ITGU esteve crescente ao longo do dia, atingindo os valores máximos nos horários das 13 e 15 h, quando voltou a decrescer. Segundo SOUZA et al. (2002), existem limites de ITGU definidos para diversas espécies de animais, como a de bovinos, pelo National Weather Service - USA, estando em análise os índices de ITGU para outras espécies de interesse zootécnico. De acordo com a citação de vários autores (CESAR et al., 2004; OLIVEIRA et al., 2005; SANTOS et al., 2006; ANDRADE et al., 2007), em trabalhos com ovinos na região semiárida nordestina, valores de ITGU acima de 78 foram considerados como fora da zona de conforto térmico para ovinos, apesar de ainda não ter classificação definitiva, principalmente com animais nativos da região. Tomando-se por base essas citações, observa-se que, às $11 ; 13$ e $15 \mathrm{~h}$, os valores do ITGU $(80,1 ; 81,3$ e 82,1, respectivamente) estiveram acima da zona de conforto térmico para ovinos.

A velocidade do vento dentro das instalações variou entre 1,0 e $1,4 \mathrm{~m} \mathrm{~s}^{-1}$; segundo McDOWELL (1989), ventos com velocidade de 1,3 a 1,9 m s s $^{-1}$ são ideais para a criação de animais domésticos, causando preocupações quando esse atinge $8,0 \mathrm{~m} \mathrm{~s}^{-1}$. Quanto à CTR, observa-se que os valores diferiram estatisticamente $(\mathrm{P}<0,05)$, com exceção dos valores das 7 e das $17 \mathrm{~h}$, que se apresentaram iguais. $\mathrm{O}$ valor das $15 \mathrm{~h}\left(543,5 \mathrm{~W} \mathrm{~m}^{-2}\right)$ foi o mais elevado, e a CTR teve crescimento ao longo do dia, até as $15 \mathrm{~h}$, quando começou a decrescer. MORAES et al. (2004), observando o efeito da época do ano sobre características termorreguladoras de caprinos, ovinos e bovinos em região semiárida, descrevem CTR na época chuvosa, para o ambiente interno das instalações, às $9 \mathrm{~h}$ de $617,4 \mathrm{~W} \mathrm{~m}^{-2}$ e às $15 \mathrm{~h}$ de $718,9 \mathrm{~W} \mathrm{~m}^{-2}$. No período seco, cita, às $9 \mathrm{~h}$, a CTR em cerca de $760,0 \mathrm{~W} \mathrm{~m}^{-2}$, e às $15 \mathrm{~h}, \mathrm{CTR}$ de $811,7 \mathrm{~W} \mathrm{~m}^{-2}$, sendo, em ambos os períodos, encontrados valores superiores aos deste trabalho.

O resumo da análise de variância das variáveis temperatura retal (TR) e frequência respiratória (FR) dos grupos genéticos nos períodos está apresentado na Tabela 3, e as médias e o desvio-padrão da temperatura retal (TR) e da frequência respiratória, em função dos grupos genéticos, estão apresentados na Tabela 4. 
TABELA 3. Resumo da análise de variância das variáveis: temperatura retal (TR) e frequência respiratória (FR). Analysis of variance summary of the rectal temperature (RT) and respiration frequency $(\mathrm{RF})$ of the animals.

\begin{tabular}{lcc}
\hline \multirow{2}{*}{ Fontes de Variação } & \multicolumn{2}{c}{ Quadrados Médios } \\
\cline { 2 - 3 } & TR & FR \\
\hline Grupo Genético & $1,704^{* *}$ & $1.813,24^{* *}$ \\
Período & $35,86^{* *}$ & $5.971,35^{* *}$ \\
Grupo genético x Turno & $0,288^{\mathrm{NS}}$ & $207,73^{\mathrm{NS}}$ \\
Erro & 0,1214 & 185,2482 \\
$\mathrm{R}^{2}$ & 0,15 & 0,03 \\
$\mathrm{C} . \mathrm{V} .(\%)$ & 0,89 & 29,43 \\
\hline$* * \mathrm{P}<0,01{ }^{\mathrm{NS}}$ - não-significativo. & &
\end{tabular}

A TR dos grupos genéticos Cariri, Barriga Negra e Cara Curta não diferiu ( $>0,05$ ) estatisticamente entre si. O Morada Nova diferiu $(\mathrm{p}<0,05)$ dos demais grupos, apresentando TR $\left(38,6{ }^{\circ} \mathrm{C}\right)$ inferior aos demais grupos, sendo esse fato justificado pelo menor porte dos animais e pelagem mais clara e refletiva, o que facilita a reflexão dos raios solares. Mesmo os valores de TA, Tgn, ITGU e CTR estando fora da ZCT, principalmente no período das 11 às $15 \mathrm{~h}$, todos os grupos genéticos conseguiram manter a TR dentro da normalidade para a espécie (CUNNINGHAN, 2004; DUKES \& SWENSON, 1996).

Os grupos genéticos Cariri e Barriga Negra não diferiram ( $p>0,05)$ estatisticamente entre si e apresentaram os maiores valores para a FR, que foram de 48,0 e 47,2 mov $\mathrm{min}^{-1}$, respectivamente, e isso pode ser atribuído ao fato de os animais apresentarem pelagem mais escura, favorecendo a absorção do calor. A menor FR (43,5 mov $\mathrm{min}^{-1}$ ) foi observada no Morada Nova, o que pode estar relacionado ao fato de que essa raça apresenta pelagem clara, característica que favorece a dissipação de calor. No período das 11 às 15 horas, os valores ambientais estavam fora da ZCT para os animais e, embora tenham mantido a TR constante, aumentaram a FR, que ficou acima da normal descrita para os ovinos (KOLB, 1980; REECE, 1996).

TABELA 4. Médias e desvios-padrão das variáveis: temperatura retal (TR), frequência respiratória (FR), em função dos grupos genéticos. Average values and standard deviation of the rectal temperature (RT), respiration frequency (FR), for genetic groups studied.

\begin{tabular}{lcc}
\hline \multicolumn{1}{c}{ Grupo Genético } & TR $\left({ }^{\circ} \mathrm{C}\right)$ & FR $\left(\mathrm{mov} \mathrm{min}^{-1}\right)$ \\
\hline Cariri & $39,0 \mathrm{a} \pm 0,3$ & $48,0 \mathrm{a} \pm 14,3$ \\
Barriga Negra & $38,9 \mathrm{a} \pm 0,3$ & $47,2 \mathrm{a} \pm 14,2$ \\
Cara Curta & $38,9 \mathrm{a} \pm 0,4$ & $46,2 \mathrm{~b} \pm 14,4$ \\
Morada Nova & $38,6 \mathrm{~b} \pm 0,4$ & $43,5 \mathrm{c} \pm 11,9$ \\
\hline *Médias nas linhas seguidas da mesma letra não diferem entre si, pelo teste de Tukey, a 5\% de probabilidade.
\end{tabular}

De acordo com ARRUDA et al. (1984), um importante mecanismo, de algumas espécies, utilizado para manter a homeotermia é a sudorese, sendo caprinos e ovinos menos dotados de glândulas sudoríparas que os bovinos, utilizando mais o processo respiratório para manter a temperatura corporal.

As médias e o desvio-padrão do índice de tolerância ao calor (ITC), da temperatura superficial (TS) e dos gradientes térmicos entre temperatura retal e temperatura superficial (TR-TS) e temperatura superficial e a temperatura do ar (TS-TA), em função dos grupos genéticos, estão apresentados na Tabela 5. Observou-se que o ITC não diferiu $(p>0,05)$ entre os grupos genéticos. Os valores médios encontrados correspondem a 10, ou próximos a esse número, que é o valor máximo encontrado para o índice, demonstrando que os animais apresentaram alta capacidade de adaptação à região, com alta tolerância ao calor. A tolerância ao calor em ovinos tem sido atribuída 
a diferenças genotípicas (GALL, 1980), o que tem sido observado também em outras espécies, como caprinos e bovinos indianos. De acordo com os resultados encontrados, os animais apresentaram FR acima da faixa normal para a espécie, mas esses mostraram-se altamente adaptados à região em estudo, demonstrando que a pressão de seleção sobre ovinos nativos em regiões semiárida, constantemente sobre índices ambientais elevados, pode ter elevado naturalmente a frequência respiratória, sem que isso, obrigatoriamente, caracterize situação de desconforto térmico.

TABELA 5. Média e desvios-padrão do índice de tolerância ao calor, temperatura superficial e dos gradientes térmicos entre temperatura retal e temperatura superficial e entre a temperatura superficial e a temperatura retal, em função dos grupos genéticos. Average values and standard deviations for the index of heat tolerance, surface temperature and thermal gradients between rectal and surface temperatures and between the surface and rectal temperatures, for to the genetic groups studied.

\begin{tabular}{lcccc}
\hline Grupo Genético & ITC & TS $\left({ }^{\circ} \mathrm{C}\right)$ & TR-TS $\left({ }^{\circ} \mathrm{C}\right)$ & TS-TA $\left({ }^{\circ} \mathrm{C}\right)$ \\
\hline Cariri & $9,8 \mathrm{a} \pm 0,2$ & $33,2 \mathrm{a} \pm 0.3$ & $5,8 \mathrm{~b} \pm 0,1$ & $4,8 \mathrm{~b} \pm 0,2$ \\
Barriga Negra & $9,9 \mathrm{a} \pm 0,4$ & $34,0 \mathrm{a} \pm 0,2$ & $4,9 \mathrm{a} \pm 0,1$ & $5,6 \mathrm{a} \pm 0,2$ \\
Cara Curta & $10,0 \mathrm{a} \pm 1,3$ & $34,0 \mathrm{a} \pm 0,2$ & $4,9 \mathrm{a} \pm 0,1$ & $5,6 \mathrm{a} \pm 0,2$ \\
Morada Nova & $10,0 \mathrm{a} \pm 0,3$ & $33,8 \mathrm{a} \pm 0,2$ & $4,8 \mathrm{a} \pm 0,1$ & $5,4 \mathrm{a} \pm 0,2$ \\
\hline TURNOS & \multicolumn{5}{l}{} \\
\hline Manhã & $33,5 \mathrm{a} \pm 0,1$ & \\
Tarde & - & $34,2 \mathrm{~b} \pm 0,1$ & \\
*Médias nas colunas seguidas de letras iguais não diferem entre si, pelo teste de Tukey, a 5\% de probabilidade.
\end{tabular}

A temperatura superficial média não teve diferença significativa $(\mathrm{p}>0,05)$ entre os grupos genéticos, corroborando os resultados obtidos por SILVA et al. (2004), em trabalhos com ovinos na região semiárida, e que também citam TS semelhante à deste trabalho. O gradiente térmico entre TR-TS revelou significância $(\mathrm{p}<0,05)$ entre o Cariri e os outros grupos genéticos, que não diferiram entre si $(\mathrm{p}>0,05)$. O Cariri apresentou gradiente térmico mais elevado, o que pode ser justificado pela sua pelagem escura e por ter maior porte.

O gradiente térmico entre TS-TA revelou significância $(\mathrm{p}<0,05)$ entre o Cariri e os demais grupos, que não diferiram entre si (p>0,05). Segundo HABEEB et al. (1992), o redirecionamento do fluxo sanguíneo e a vasodilatação facilitam a dissipação do calor por mecanismos não-evaporativos, entretanto a eficácia desses mecanismos depende do gradiente térmico entre o corpo do animal e o ambiente.

Os grupos genéticos Cariri e Barriga Negra apresentaram valores semelhantes para a TR e a FR, sendo o gradiente entre a TR e a TS superior no Cariri, e o gradiente entre a TS e a TA inferior, demonstrando que o Cariri foi menos eficiente em realizar a vasodilatação periférica, onde a diferença entre a temperatura de sua pele e a temperatura ambiente estava mais próxima. $\mathrm{O}$ grupo genético Morada Nova apresentou as menores TR e FR, em relação aos demais grupos. Pelas exposições, o Morada Nova pode ser considerado o mais adaptado às condições ambientais adversas, e o grupo genético Cariri, principalmente pelas variáveis fisiológicas, como também características fenotípicas (Figura 1), como o menos adaptado nas condições experimentais.

\section{CONCLUSÕES}

A temperatura ambiente, a temperatura do globo negro, o índice de temperatura do globo negro e umidade e a carga térmica de radiação ficaram dentro das instalações, no período das 11 às $15 \mathrm{~h}$, acima da zona de conforto térmico para ovinos adultos. 
A umidade relativa do ar no interior das instalações, das 9 às 17 h, ficou dentro da zona de conforto térmico para ovinos adultos.

Apesar das condições ambientais elevadas, os animais conseguiram manter a temperatura retal dentro da normalidade, havendo aumento na frequência respiratória.

Os animais apresentaram-se bem adaptados às condições ambientais da região, pois ofereceram alto grau de adaptabilidade, medido por meio do índice de tolerância ao calor.

O Morada Nova foi o que apresentou menor temperatura retal e frequência respiratória em relação aos demais grupos, podendo ser considerado o mais adaptado às condições experimentais. $\mathrm{O}$ Cariri, apesar de ter valores semelhantes ao Barriga Negra para temperatura retal e frequência respiratória, apresentou piores desempenhos nos gradiente térmicos, podendo ser considerado o menos adaptado.

\section{REFERENCIAS}

ANDRADE, I.S.; SOUZA, B.B.; PEREIRA FILHO, J.M.; SILVA, A.M.A. Parâmetros fisiológicos e desempenho de ovinos Santa Inês submetidos a diferentes tipos de sombreamento e à suplementação em pastejo. Ciência e Agrotecnologia, Lavras, v.31, n.2, p.540-7, 2007.

ARRUDA, F.A.V.; FIGUEIREDO, E.A.P.; PANT, K.P. Variação da temperatura corporal de caprinos e ovinos sem-lã em Sobral. Pesquisa Agropecuária Brasileira, Brasília, v.19, n.7, p. 91519, 1984.

BAÊTA, F.C.; SOUZA, C. Ambiência em edificações rurais: conforto animal. Viçosa: UFV, 1997. $246 \mathrm{p}$.

BACARI JÚNIOR, F.; POLASTRE, R.; FRÉ, C.A.; ASSIS, P.S. Um novo índice de tolerância ao calor para bubalinos. Correlação com o ganho de peso. IN: REUNIÃO ANUAL DA SOCIEDADE BRASILEIRA DE ZOOTECNIA, 28., 1986. Campo Grande. Anais... Campo Grande: SBZ, 1986. p.274.

BACARI JÚNIOR, F. Métodos e técnicas de avaliação da adaptabilidade dos animais às condições tropicais. In: SIMPOSIO INTERNACIONAL DE BIOCLIMATOLOGIA ANIMAL NOS TRÓPICOS: PEQUENOS E GRANDES RUMINANTES, 1., 1990, Sobral. Anais... Sobral: EMBRAPA/CNPC, 1990. p.9-17.

BERBIGIER, P. Effect of heat on intensive meat production in the tropics: cattle, sheep and goats, pigs. IN: CICLO INTERNACIONAL DE PALESTRAS SOBRE BIOCLIMATOLOGIA ANIMAL, 1., 1989, Botucatu. Anais... Jaboticabal: FMVZ/UNESP/FUNEP, 1989. p.7-44.

BIANCA, W.; KUNZ, P. Physiological reactions of three breeds of goats to cold, heat an hight altitude. Livestock Production Science, Amsterdam, v.5, n.1, p.57-69, 1978.

BOND, T.E.; KELLY, C.F.; ITTER, N.R. Radiation studies of painted shade materials. Transaction of the ASAE, St. Joseph, v.35, n.6, p.389-92, 1954.

BUFFINGTON, C.S.; COLLAZO-AROCHO, A.; CANTON, G.H.; PITT, D. Black globe humidity comfort index for dairy cows. St. Joseph: ASAE, 1977. 19 p.

CESAR, M.F.; SOUZA, B.B.; SOUZA, W.H.; PIMENTA FILHO, E.C.; TAVARES, G.P.; MEDEIROS, G.X. Avaliação de parâmetros fisiológicos de ovinos Dorper, Santa Inês e seus mestiços perante condições climáticas do trópico semiárido Nordestino. Ciência e Agrotecnologia, Lavras, v.28, n.3, p.614-20, 2004.

CHIMINEAU, P. Médio ambiente y reproducción animal. World Animal Review, Roma, v.77, n.1, p.2-14, 1993.

CUNNINGHAM, J.G. Tratado de fisiologia veterinária. 3.ed. Guanabara Koogan, 2004. 596 p. 
DUKES, H.H.; SWENSON, H.J. Fisiologia dos animais domésticos. 11.ed. Rio de Janeiro: Guanabara Koogan, 1996. 856 p.

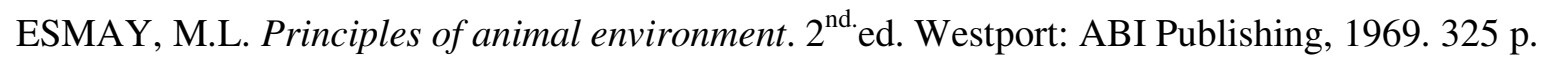

GALL, C. Relationship between body conformation and production in dairy goats. Journal of Animal Science, Indianapolis, v.63, n.10, p.1.768-81, 1980.

HABEEB, A.L.M.; MURRAY, L.F.M.; KAMAL, T.H. Farm animals ant the environment. Cambridge: CAB, 1992. 428 p.

KOLB, E. Coração e circulação. In: GÜNTLER, H.; KETZ, H.A.; KOLB, L.; SCHRÖDER, H.; SEIDEL, H. Fisiologia veterinária. 4.ed. Rio de Janeiro: Guanabara Koogan, 1980, p.263-94

LU, C.D. Effects of heat stresses on goat production. Small Ruminants Research, Amsterdam, v.2, p.151,62, 1989.

McDOWELL, R.E. Bases biológicas de la producción animal en zonas tropicales. São Paulo: Ícone, 1989. $183 \mathrm{p}$.

MONTY JUNIOR, D.E.; KELLY, L.M.; RICE, W.R. Acclimatization of St Croix, Karakul and Rambouillet sheep to intense and dry summer heat. Small Running Research, Amsterdam, v.4, n.4, p.379-92, 991.

MORAES, D.A.E.F.; BENTO, C.A.P.; SOUZA JÚNIOR, S.C.; VASCONCELOS, A.M.; SOUZA, W.G. Efeito da época do ano sobre características termorreguladoras de caprinos, ovinos e bovinos na região semiárida. In: CONGRESSO NORDESTINO DE PRODUÇÃO ANIMAL, 3., 2004, Campina Grande. Anais ... Campina Grande: Universidade Federal de Campina Grande, 2004. 1 CD-ROM.

OLIVEIRA, F.M.M.; DANTAS, R.T.; FURTADO, D.A.; NASCIMENTO, J.W.B.; MEDEIROS, A.N. Parâmetros de conforto térmico e fisiológicos de ovinos Santa Inês, sob diferentes sistemas de acondicionamento. Revista Brasileira de Engenharia Agrícola e Ambiental, Campina Grande, v.9, n.4, p.631-5, 2005.

REECE, W.O. Respiração nos mamíferos. In: DUKES, H. H.; SWENSON, M. J. Fisiologia dos animais domésticos. 10.ed. Rio de Janeiro: Guanabara Koogan, 1996. p.199-205.

SANTOS, F.C.B.; SOUZA, B.B.; ALFARO, C.H.P.; CEZAR, M.F.; PIMENTA FILHO, E.C.; ACOSTA, A.A.A.; SANTOS, J.R.S. Adaptabilidade de caprinos exóticos e naturalizados ao clima semiárido do Nordeste brasileiro. Ciência e Agrotecnologia, Lavras, v.29, n.1. p.142-9, 2005.

SANTOS, J.R.S.; SOUZA, B.B.; SOUZA, W.H.; CEZAR, M.F.; TAVARES, G.P. Respostas fisiológicas e gradiente térmicos de ovinos das raças Santa Inês, Morada Nova e seus cruzamentos com a raça Dorper as condições do semiárido nordestino. Ciência e Agrotecnologia, Lavras, v.30, n.5, p.995-1001, 2006.

SAS INSTITUTE. Statistical Analysis System: release 6.08 (software). Cary, 1999. 620 p.

SILVA, R.G. Introdução à bioclimatologia animal. São Paulo: Nobel, 2000. 286 p.

SILVA, G.A.; SOUZA, B.B.; ALFARO, C.E.P.; SILVA, E.M.N.; AZEVEDO, S.A.; NETO, J.A.; SILVA, R.M.N. Efeito da época do ano sobre os parâmetros fisiológicos de caprinos no semiárido. In: SIMPÓSIO DE CONSTRUÇÕES RURAIS E AMBIÊNCIA, 1., 2004, Campina Grande. Anais... Campina Grande: Universidade Federal de Campina Grande, 2004.

SOUZA, C.F.; TINÔCO, I.F.F.; BAÊTA, F.C.; FERREIRA, W.P.M.; SILVA, R.S. Avaliação de matérias alternativos para confecção do termômetro de globo. Ciência e Agrotecnologia, Lavras, v.26, n.1, p.157-64, 2002. 